EGU2020-21882

https://doi.org/10.5194/egusphere-egu2020-21882

EGU General Assembly 2020

(c) Author(s) 2020. This work is distributed under

the Creative Commons Attribution 4.0 License.

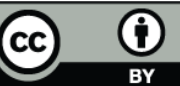

\title{
Research products across space missions - a prototype for central storage, visualization and usability
}

Mario D'Amore, Andrea Naß, Martin Mühlbauer, Torsten Heinen, Mathias Boeck, Jörn Helbert, Torsten Riedlinger, Ralf Jaumann, and Guenter Strunz

DLR, Planetary Research, Berlin, Germany (mario.damore@dlr.de)

For planetary sciences, the main archives to archived access to mission data are ESA's Planetary Science Archive (PSA) and the Planetary Data System (PSA) nodes in the USA. Along with recent and upcoming planetary missions the amount of different data (remote sensing/in-situ data, derived products) increases constantly and serves as basis for scientific research resulting in derived scientific data and information. Within missions to Mercury (BepiColombo), the Outer Solar System moons (JUICE), and asteroids (NASA`s DAWN), one way of scientific analysis, the systematic mapping of surfaces, has received new impulses, also in Europe. These systematic surface analyses are based on the numeric and visual comparison and combination of different remote sensing data sets, such as optical image data, spectral-/hyperspectral sensor data, radar images, and/or derived products like digital terrain models. The analyses mainly results in map figures, data, and profiles/diagrams, and serves for describing research investigations within scientific publications.

Handling these research products equivalently to missions' base data in the main archives, webbased geographic information systems became a common mean to impart spatial knowledge to all kinds of possible users in the last years. So, further platforms and initiatives came up handling planetary data within web-based GIS, services, or/and virtual infrastructures. Those systems are either built upon proprietary software environments, but more common upon a well-established stack of open source software such as PostgreSQL, GeoServer (server for sharing geospatial data) and a graphical user interface based on JavaScript. Applicable standards developed by the Open Geospatial Consortium (OGC), such as the Web Map Service (WMS) and the Web Feature Service (WFS) server-based data storage as interface between the user interface and the server.

This contribution aims to a prototypical system for the structured storage and visualization of planetary data compiled and developed within or with the contribution of Institute for Planetary Research (PF, DLR). Consequently, it enables user groups to store and spatially explore research products centrally, sustainably across multiple missions and scientific disciplines [1]. 
Technically, the system is based on two components: 1) an infrastructure that provides data storage and management capabilities as well as OGC-compliant interfaces for collaborative and web-based data access services, such as the EOC Geoservice [2]. 2) UKIS (Environmental and Crisis Information Systems), a framework developed at DFD for the implementation of geoscientific web applications [3]. Substantially the prototype based on a recent approach developed within PF [4] where an existing database established at Planetary Spectroscopy Laboratory (PSL), handling different kind of spatial data, meets a vector-based data collection of thematic, mainly geologic and geomorphologic mapping results [5].

An information system of this kind is essential to ensure the efficient and sustainable utilization of the information already obtained and published. This is considered a prerequisite for guaranteeing a continuous and long-term use of scientific information and knowledge within institutional frameworks.

[1] Naß, et al (2019) EPSC \#1311

[2] Dengler et al. (2013) PV 2013, elib.dlr.de/86351/

[3] Mühlbauer (2019) dlr.de/eoc/UKIS/en/

[4] Naß, d 'Amore, Helbert (2017) EPSC \#646-1

[5] Naß, Dawn Science Team (2019) EPSC \#1304 